Musées, Patrimoine et Culture scientifiques et techniques

$181 \mid 2019$

janvier-février 2019

\title{
Repenser les formations doctorales. De la communication efficace à la communication incarnée
}

\section{Mélodie Faury et Lionel Maillot}

\section{(2) OpenEdition}

Édition électronique

URL : http://journals.openedition.org/ocim/2138

DOI : $10.4000 /$ ocim. 2138

ISSN : 2108-646X

Éditeur

OCIM

\section{Édition imprimée}

Date de publication : 1 janvier 2019

Pagination : 12-21

ISSN : 0994-1908

\section{Référence électronique}

Mélodie Faury et Lionel Maillot, « Repenser les formations doctorales. De la communication efficace à

la communication incarnée », La Lettre de I'OCIM [En ligne], 181 | 2019, mis en ligne le 01 janvier 2020, consulté le 17 mars 2020. URL : http://journals.openedition.org/ocim/2138 ; DOI : https://doi.org/

10.4000/ocim.2138

Ce document a été généré automatiquement le 17 mars 2020

Tous droits réservés 


\section{Repenser les formations doctorales. De la communication efficace à la communication incarnée}

Mélodie Faury et Lionel Maillot

Nuit Européenne des Chercheurs à Dijon

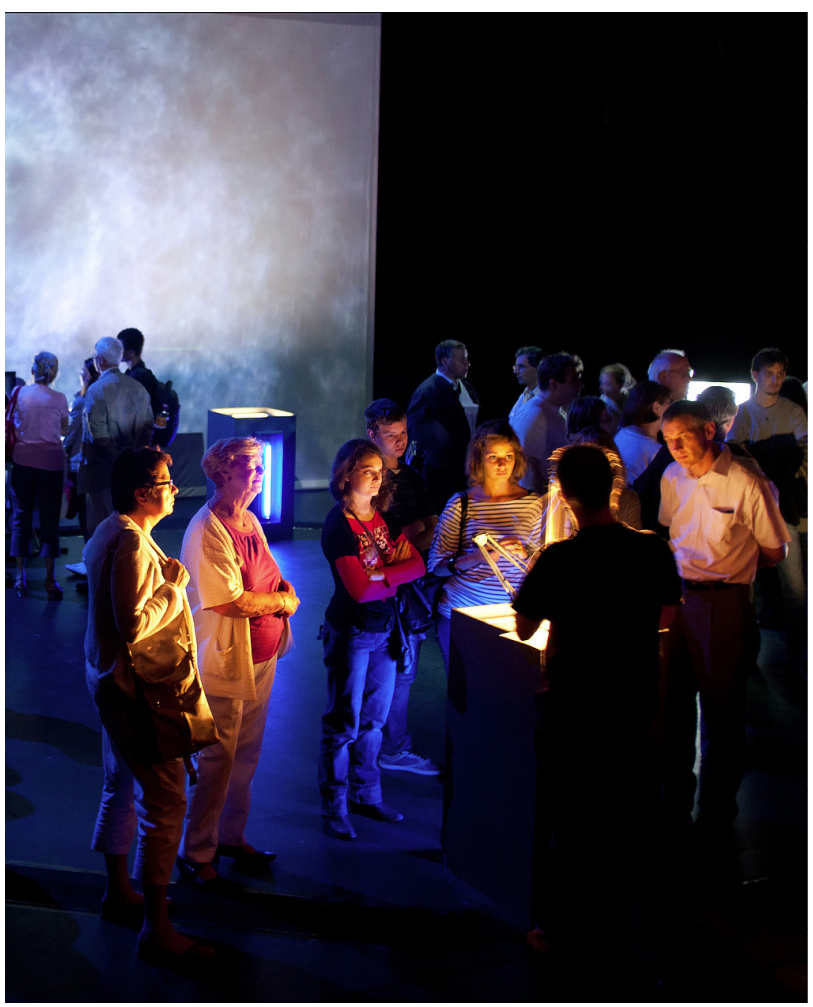

(C) Vincent Arbelet 


\section{Former les doctorants}

1 Impliquant des professionnels de centres de culture scientifique, d'associations, de musées, d'entreprises ou des personnels universitaires, les écoles doctorales françaises proposent des formations à la communication pour leurs doctorants. Ces formations sont diverses et recouvrent le champ de la culture scientifique ${ }^{1}$. Elles peuvent ou non comporter une phase de pratique, comme par exemple la participation à une action de communication scientifique ou à un dispositif science-société (voir les formations au dispositif Ma thèse en 180 secondes, Experimentarium ou encore Accompagnement en Sciences et Technologie à l'École Primaire, Astep).

2 Nous réalisons ce type de formations en tant que professionnels de la CSTI et des projets science-société. En tant que chercheurs travaillant tous les deux à l'intersection des sciences de l'information et de la communication et des études de sciences, nous engageons également une réflexion sur le déroulement et les enjeux de ces formes de communication des doctorants. Ainsi, tirant des enseignements de travaux qui, notamment, remettent en cause un modèle dominant de communication (le « modèle déficitaire »), nous proposons de repenser ces formations doctorales.

\section{Une influence théorique, source de questionnements pratiques}

3 Depuis, la fin des années 1990, plusieurs études reprennent une observation initiée par Baudouin Jurdant en 1970 quant à l'existence d'un modèle de communication prégnant : le modèle déficitaire (ou " deficit model $»)^{2}$. Lorsque ce modèle est sous-jacent, la communication "grand public » a pour but de combler le déficit de connaissance ou de confiance séparant les savants et les profanes. Ce modèle se structure sur le principe émetteur-message-récepteur.

Les formes de communication de type "présentation » ou " explication didactique ", généralement associées à ce modèle, présentent des atouts : une exposition claire d'un savoir scientifique constitue une information qui peut être utile aux citoyens. Ces formes de communication sont largement employées avec un public captif, ignorant de ce qui va lui être enseigné, et qui est donc là pour apprendre, comme c'est souvent le cas en classe.

Dans le champ de la communication publique des sciences, de la vulgarisation ou culture scientifique, les situations des publics peuvent être diverses. Plusieurs études montrent qu'un positionnement dans une posture déficitaire n'est pas sans conséquences quant aux postures mobilisées et aux liens sciences-société construits ${ }^{3}$. En effet, le modèle déficitaire a largement été critiqué depuis plus de 30 ans, parce qu'il serait associé à une conception positiviste de la science ; pour son inefficacité (plusieurs études ont remis en cause les résultats de politiques publiques visant à augmenter la connaissance scientifique) ; pour la posture de "sachant " et le sentiment d'infantilisation qu'il peut générer ; enfin, parce qu'il véhicule l'idée d'une réception passive de l'information par le public. 
"Cratères et météorites ", une formation pour les doctorantes.

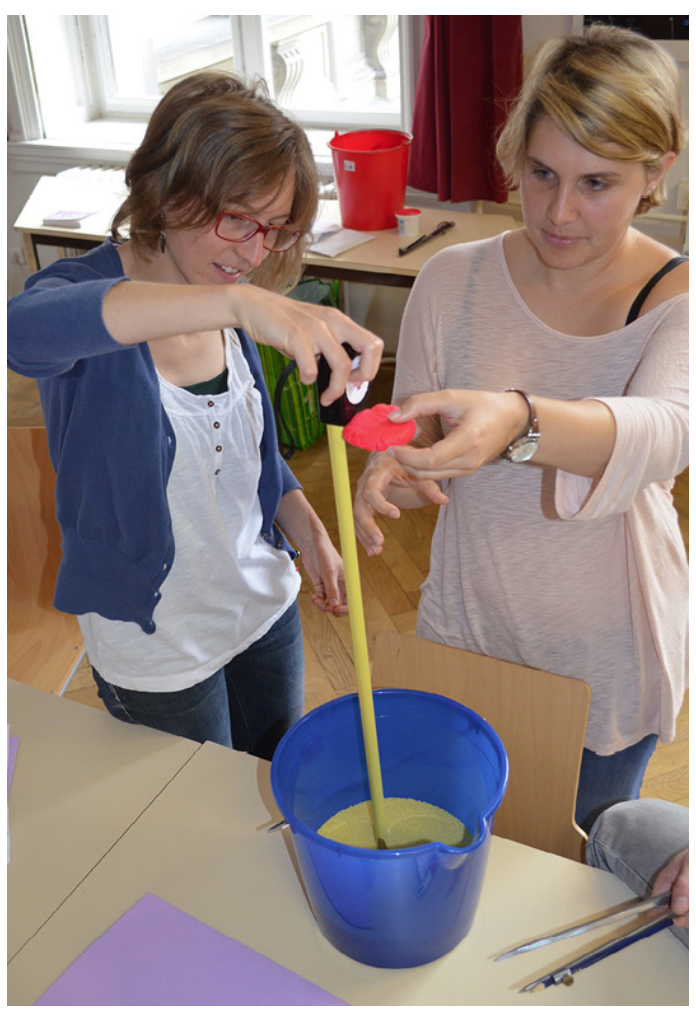

(c) Maison pour la science en Alsace

6 Au-delà de la critique du modèle déficitaire, plusieurs travaux questionnent son utilisation implicite et le plus souvent non-interrogée, parmi une multitude d'autres modèles de communication possibles. Les conséquences d'un manque de conscience de l'emprunt de cette posture " par défaut » dans les actions de culture scientifique sont pointées. Retenons deux travaux concernant les chercheurs, puis les formateurs de chercheurs :

7 - dans sa thèse ${ }^{4}$, Cyrille Bodin décrit un effet " culbuto » : malgré les tentatives renouvelée de le mettre à l'horizontal, le culbuto est ce jouet qui revient toujours à la verticale. Lors d'actions de vulgarisation dans des villages des sciences, ou lors de conférences citoyennes, les chercheurs observés par C. Bodin reviennent à une posture top-down alors que, dans leur intention initiale, leur action de communication est voulue comme symétrique. Ainsi, les programmes ou les structures peuvent souhaiter ou revendiquer des postures de dialogue, mais dans la pratique une forme de communication unilatérale reprend le dessus ;

8 - du coté des formateurs, les américains Besley et Dudo ont interviewé des formateurs de chercheurs. Alors que ces formateurs sont en accord avec l'intention qui consiste à entrer dans une dynamique de dialogue entre scientifiques et citoyens, dans les faits, le coaching qu'ils proposent ancre les stagiaires dans un modèle de communication déficitaire. Finalement "l'objectif est d'aider les chercheurs à exposer clairement leur recherche pour augmenter la connaissance du public $»^{5}$.

9 En tant que formateurs de doctorants, nous ne pouvons prétendre être prémunis de l'effet " culbuto ». D'autant plus que les compétences classiquement visées des formations mobilisent des termes du modèle « Émetteur-Message-Récepteur ». Lorsque 
la formation propose d'apprendre à mieux « diffuser » ou « transmettre » un message, la terminologie associée est unilatérale dans le rapport entre les protagonistes : parlant d'action "face " public, d'un public " cible ", de message " impactant ", ou encore recourant à des bonnes recettes qui s'appuient sur des leviers comme « l'adaptation du discours au public ». Ce public reste une cible, un récepteur. Au mieux, quelques " feedback » servent à réguler la diffusion d'un message ${ }^{6}$ de l'un (le récepteur) vers l'autre (l'émetteur) sans véritable prise en compte de l'intérêt et de l'effet d'un dialogue.

Ces réflexions nous ont donc invités à questionner nos pratiques, de manière critique, à repérer nos attitudes " déficitaires ", à discuter les présupposés de nos formations et à identifier le modèle de communication structurant que nous utilisons parfois sans y penser. Nous souhaitons partager ce mouvement réflexif et ses effets, sans pour autant exclure les points aveugles qui immanquablement perdurent.

11 Dans nos explorations, nous avons notamment mis au jour des enjeux et des objectifs de formation distincts de celui de l' " efficacité » de la communication, et qui s'affranchit du point de vue réducteur de la « réception d'un message ».

\section{La découverte des « à-côtés »}

12 À l'étude de ces travaux théoriques, nous joignons donc une démarche d'observation réflexive de nos formations. Il nous semble en effet que le mouvement de la communication efficace délaisse des situations, des phénomènes et des enjeux majeurs pour la formation des chercheurs. Ce sont précisément ces " à-côtés " que nous proposons de remettre au centre, pour revenir à un ancrage dans l'expérience vécue et dans la dynamique réflexive. Engager les doctorantes et les doctorants à parler de manière située, depuis leur propre vécu, parcours, place ${ }^{7}$.

Échanges conviviaux lors de l'école interdisciplinaire : parler un langage commun?

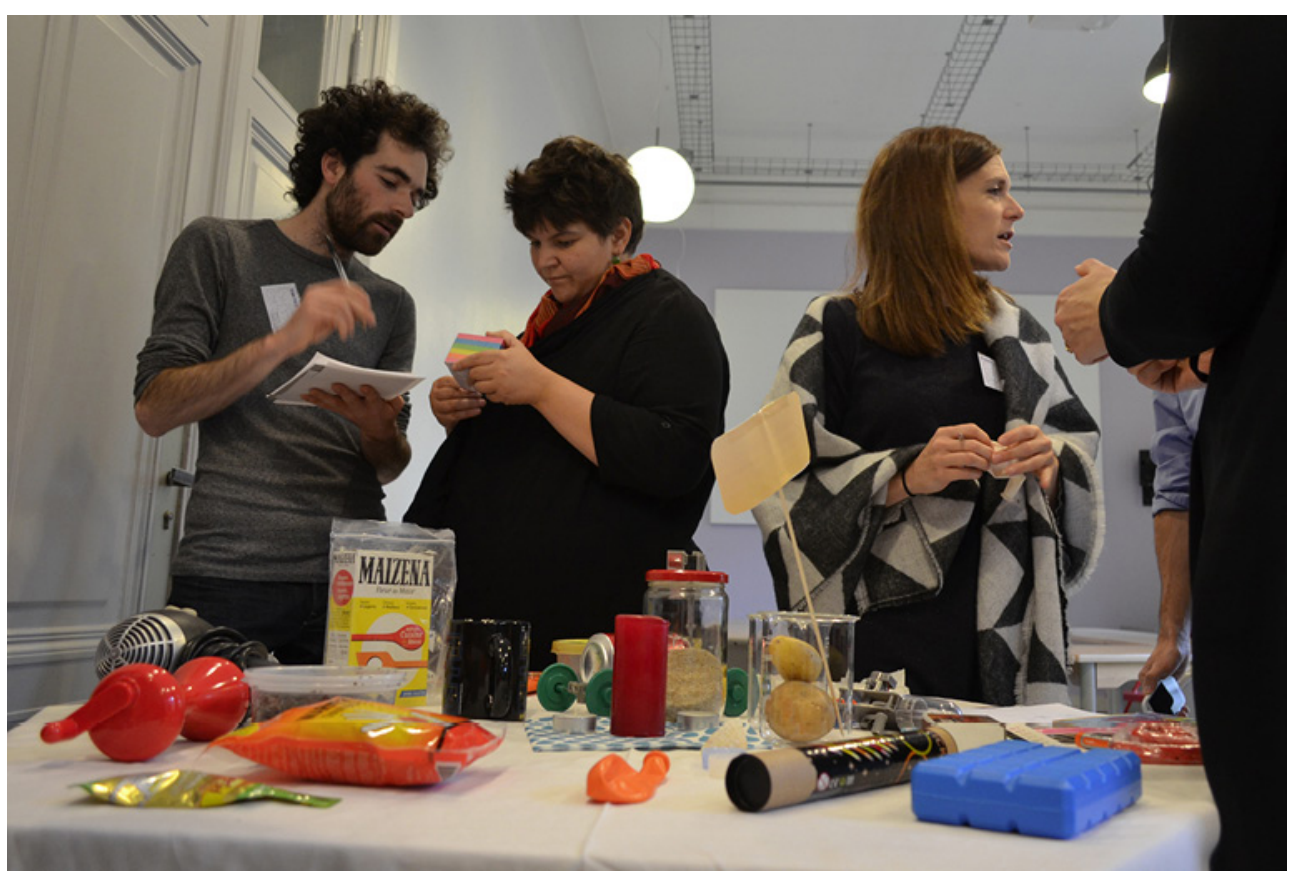

(c) Maison pour la science en Alsace 


\section{De la marge vers le centre : le cœur de nos formations} d'échange collectif et de partage d'une condition : la condition de doctorant. Qu'elles se déroulent sur une journée ou sur des temps plus longs associés à des mises en situation, elles se construisent autour de moments de pauses, de discussions informelles ou encore de repas voire de déplacements vécus ensemble. L'Experimentarium, programme associant une formation de jeunes chercheurs à des rencontres avec des publics, organise - par exemple - des déplacements dans des lycées éloignés qui impliquent la sortie en groupe de six à huit chercheurs : un minibus est loué, l'internat est réservé pour la nuitée et les doctorants déjeunent ensemble.

Dans un premier temps, en tant que formateurs de doctorants à Lyon, Dijon et Strasbourg, nous avons été stupéfaits par la rapidité avec laquelle les doctorants se saisissent de ces moments informels pour échanger sur leur vécu. Nous l'analysons comme un véritable besoin "d'en parler ». Plusieurs études observent des sentiments de solitude prégnants chez les doctorants. Une enquête réalisée en 2015 auprès de doctorants en Sciences humaines et sociales met en exergue ce verbatim cinglant : « tu te lèves le matin et tu n'existes pour personne $\|^{8}$. Le sentiment est lié à la place du doctorant dans la société, mais aussi au sein de son laboratoire et en lien avec son apprentissage professionnel : il découvre une réalité professionnelle parfois différente de ses représentations initiales, il compose avec l'inconnu, les échecs et les impasses inhérentes à toute activité de recherche. L'expérience de la pratique de recherche est source de conflits de normes et de valeurs chez les doctorants, et la relation au directeur de thèse, notamment, est centrale dans ce vécu (en positif comme en négatif). Cette dernière peut difficilement être racontée au sein même du laboratoire. L'intensité de l'expérience professionnelle induit le besoin d'« en parler » (échanger, se confier, comparer, se rassurer...) à des pairs, c'est-à-dire à d'autres doctorants. Dans un contexte de solitude et de doutes, les formations doctorales sont l'occasion de trouver des alter-égo, des "second-soi " qui sont à la fois différents (car les stagiaires proviennent souvent de disciplines diverses), mais qui ont un vécu commun : celui de l'initiation à la recherche et de cette condition de doctorant. 
La démarche d'investigation : travailler collectivement sur des dispositifs techniques disponibles en classes. Une action réalisée par des doctorants dans un collège pilote.

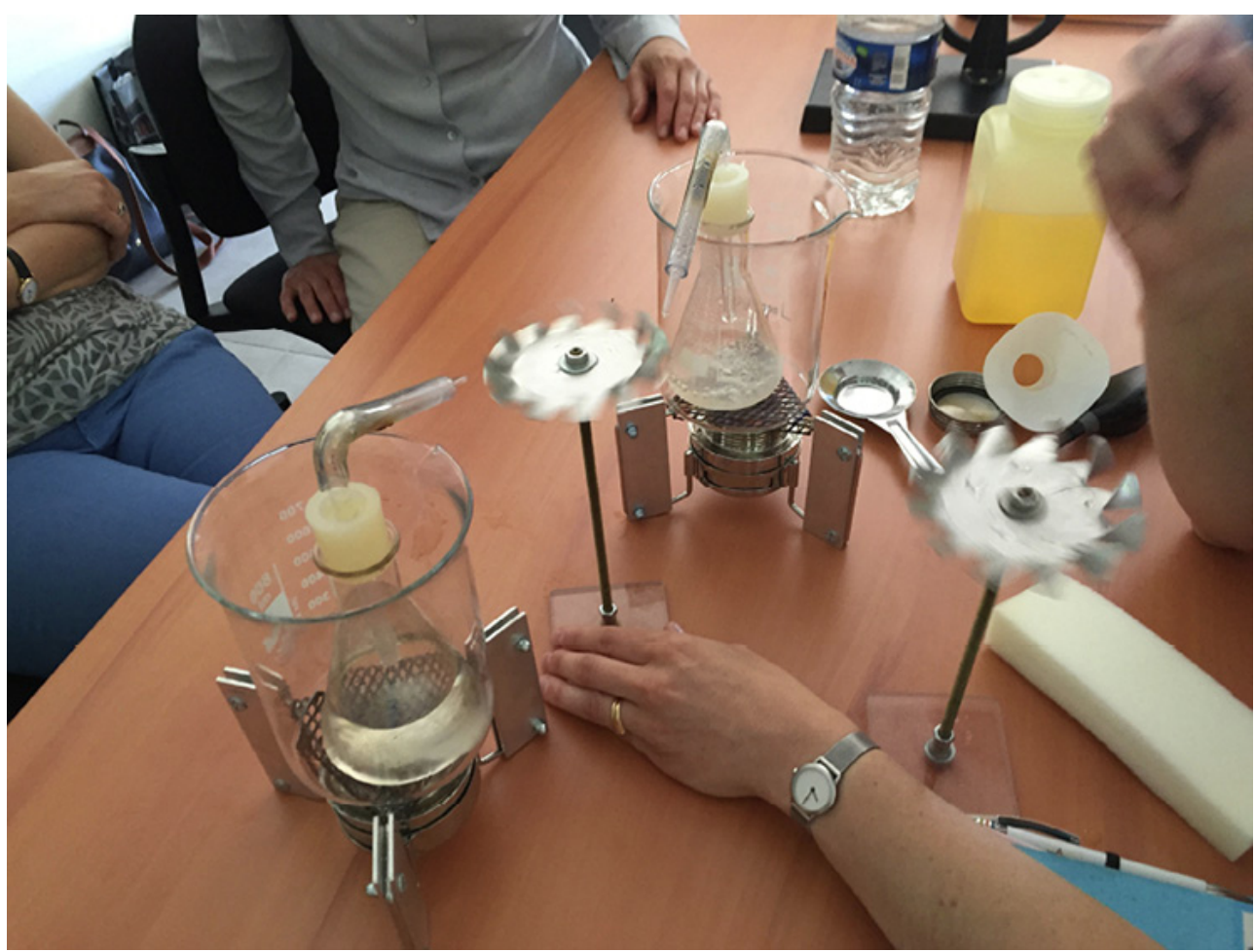

(c) Maison pour la science en Alsace

Concernant la formation Experimentarium, Mélanie dit que "ça rend la recherche plus conviviale »; Clémentine écrit : « on dit souvent de la thèse que c'est comme une grossesse, et bien pour moi l'Experimentarium c'est comme la péridurale, ça rend le travail moins douloureux !» (paroles de doctorantes à l'occasion des formations Experimentarium).

Certaines formations courtes ou associées à des programmes longs ou récurrents, qui intègrent une dimension de groupe, jouent ainsi le rôle d'espace de réconfort pour les doctorants. Compte tenu de leur richesse et de leur densité, il nous semble important de considérer les moments de parole et d'échange, de complicité, qui étaient a priori des " à-côtés ", comme étant au contraire des éléments fondamentaux et centraux de la formation. Ce peut même devenir explicitement l'objectif principal d'une formation, comme c'est le cas par exemple à Strasbourg depuis 2017-2018 : «Parler de sa thèse en traversant les frontières entre les disciplines $"$.

Mais, dans nos formations, nous insistons pour que l'attention à la place de la parole des doctorants reste centrale pour les formateurs quels que soient l'objet et les objectifs de la formation doctorale. Cet enjeu prend tout son sens dès lors qu'il s'agit de communiquer, de " parler la science ». Nous ne voyons pas, en effet, l'intérêt de former les doctorants à " parler tout seul ». Nous considérons la communication comme une rencontre qui va permettre au chercheur d'apprendre de l'autre, par l'autre et par la situation, par le contexte de médiation ou de vulgarisation. Dans la formation, les jeunes chercheurs s'exercent à cette dynamique avec l'autre. Cet autre peut être d'abord un autre "proche ", comme un autre doctorant ou une autre doctorante en formation, mais il peut s'agir ensuite, et dans un même mouvement, d'un autre " plus distant », dans son vécu, dans ses actualités : le « public ». 

pour une communication efficace. Par la rencontre entre doctorants, elles préparent la rencontre avec les publics. On parle « avec » et non plus « à » quelqu'un. On inclut l'autre, on ne le « vise » pas.

Une fois la formation réalisée, Alix, médiatrice-formatrice prend du recul vis-à-vis de la rencontre chercheur-public, mais reste présente pour aider ou conseiller.

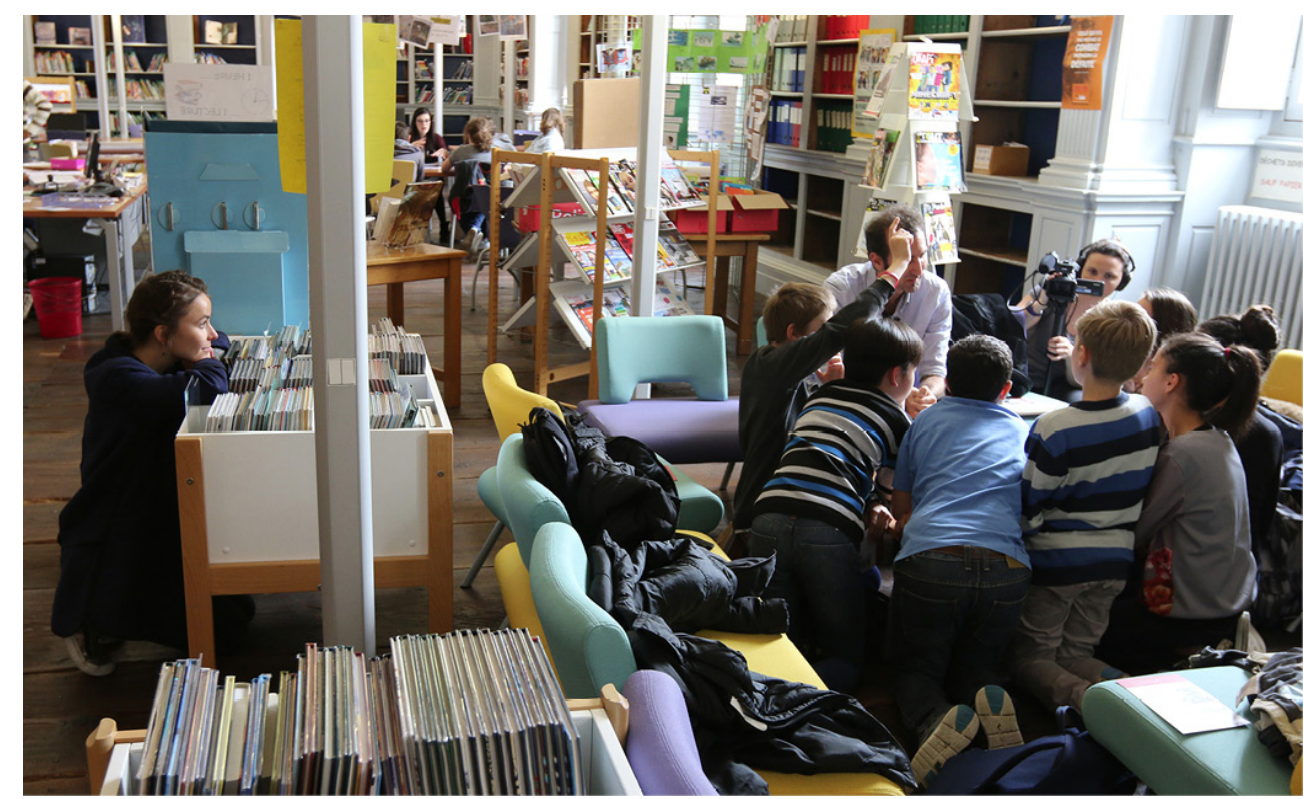

(c) Lionel Maillot

\section{Qui parle ? Les « à-côtés » de la science au cœur de la formation}

Mais de quoi parlent les doctorants entre eux ? Parlent-ils de science ? Ils partagent leur vécu de l'incertain, le doute et le questionnement permanent, les ajustements aux valeurs disciplinaires et institutionnelles, les contingences humaines et les relations professionnelles. Ils parlent spontanément à partir de leur vécu. Ils se permettent de parler ainsi et en leur nom dans les espaces et moments informels que nous ouvrons en formation. Ils parlent en tant qu'individu, utilisant le « je », avant de parler en tant que scientifiques, en tant que représentants de la science ou de leur discipline, utilisant le " on » ou le « nous ». Ils parlent de « leur science», c'est-à-dire de leur vécu particulier et contextualisé (dans un laboratoire, dans une équipe, dans une discipline particulière) de la pratique professionnelle de la science.

La posture déficitaire, à l'opposé de laquelle nous nous positionnons en tant que formateurs, privilégie la communication de messages qui sont des savoirs scientifiques établis : des "faits scientifiques ». Dans le cadre de l'enseignement scientifique, et des logiques de vulgarisation proches ou associées, on complète l'actualisation des savoirs avec des mises en situation visant à connaître, appréhender voire faire vivre la démarche scientifique (dite "démarche d'investigation ", " démarche de recherche ", ou encore " démarche expérimentale »). Cette démarche est souvent lissée, voir 
idéalisée par souci didactique ou pédagogique. Ainsi, les messages construits dans ces situations de communication, médiation ou vulgarisation scientifique gomment ce dont les doctorants parlent, « d'eux-mêmes », quand ils se le permettent, c'est-à-dire quand ils ne sont pas en représentation.

21 Il nous semble essentiel, d'un point de vue humain, et pertinent, du point de vue de la communication scientifique de ne pas effacer l'expérience vécue des (jeunes) chercheurs dans les dispositifs de communication, pour au moins deux raisons : l'intérêt qu'il y a à témoigner de la " science en train de se faire " pour la construction des relations science-société ; et la posture réflexive, individuelle et collective, que cette approche induit chez les doctorants.

Jennifer, doctorante, raconte sa recherche sur des cellules du système immunitaire, lors d'un Experimentarium sur le marché de Dijon.

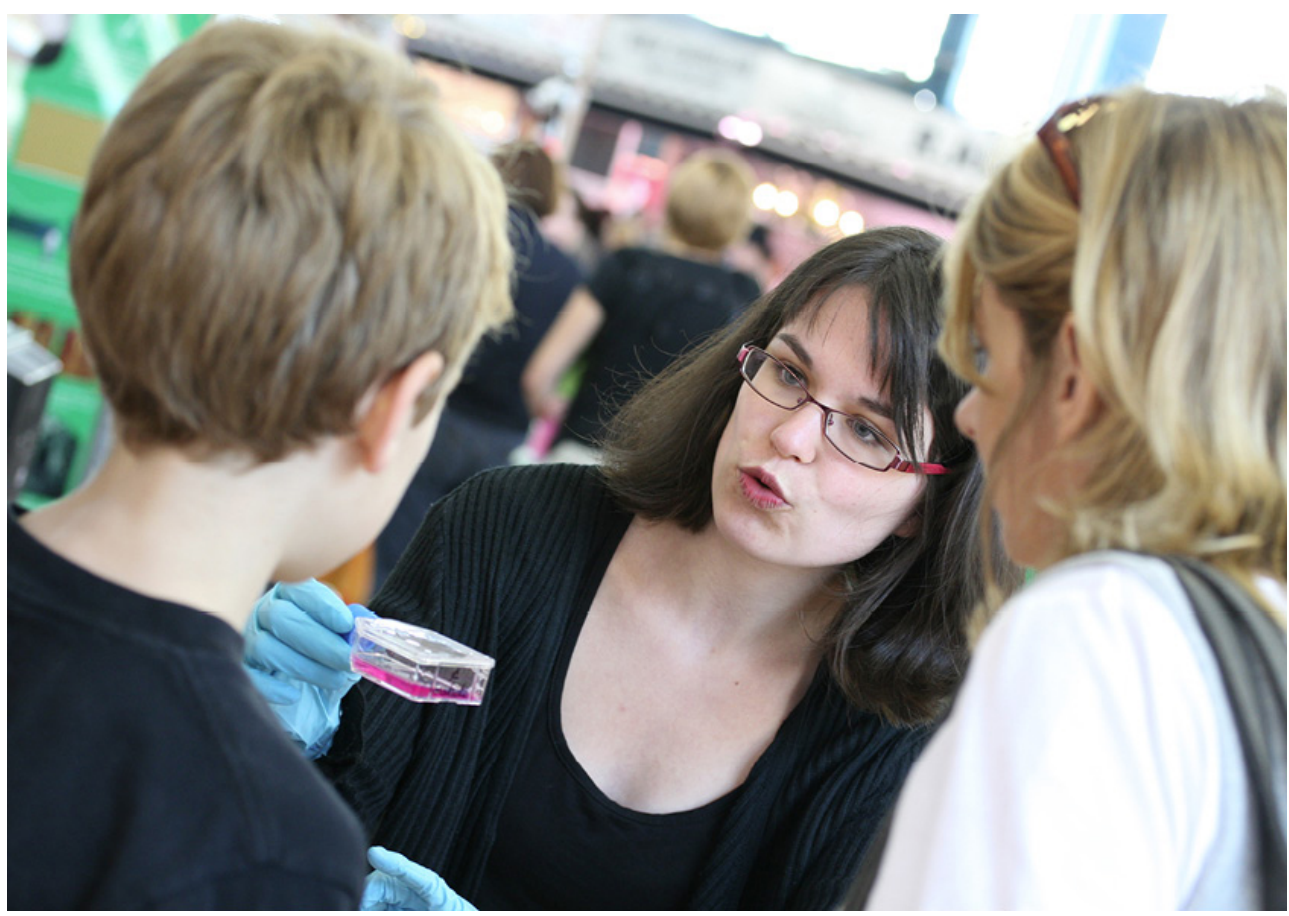

(c) Lionel Maillot 
Lors d'un Experimentarium, Nathalie, doctorante, présente son cahier de labo. Une occasion de livrer son vécu, y compris ses ratures et erreurs.

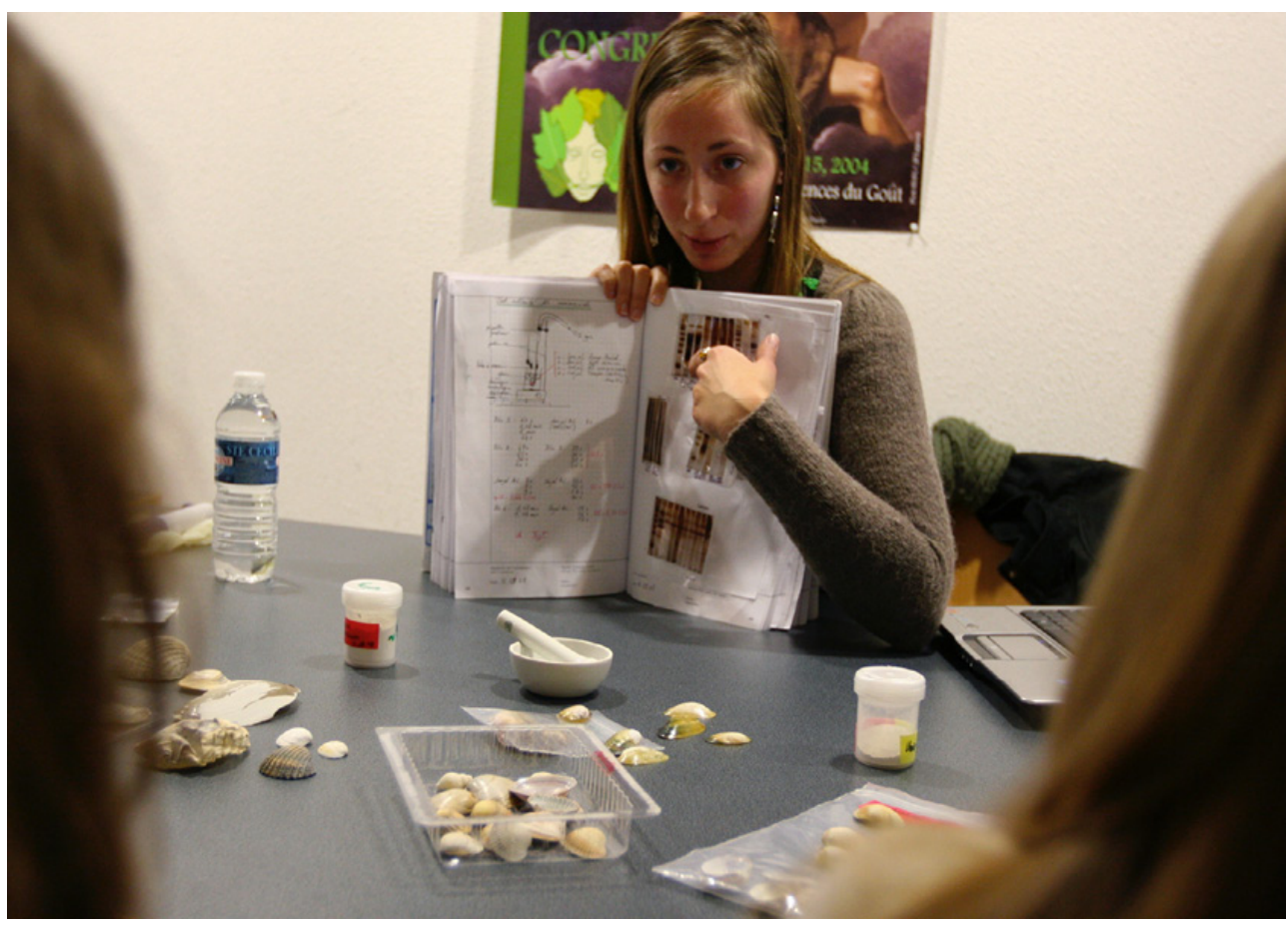

(C) Lionel Maillot

\section{De la « science en train de se faire " à l'expérience vécue de la recherche}

Le concept de "science en train de se faire ", né dans les années 1980, vient de la nécessité d'observer et de penser de façon nouvelle la production scientifique. Concernant la communication publique des sciences, l'appropriation de ce concept a amené les acteurs à sortir d'une présentation de la " science tout faite » et à assumer les inter-relations science-société, non seulement en les présentant, mais en les provoquant par l'ouverture des situations de communication à une possibilité de dialogue critique. Faisant le point sur le concept, en 2016, Antoine Blanchard remarque qu'il est bien souvent loin d'être atteint. Malgré un affichage sous le terme « science en train de se faire ", on retrouve encore un retour à la posture déficitaire. Les pratiques tendent à présenter une démarche scientifique mise en scène, lissée, qui ne prend pas en compte par exemple les contingences sociales de la vie scientifique telles que "l'incertitude intrinsèque au travail scientifique " ou encore " les traductions permettant d'enrôler les alliés pour clore les controverses "॥.

Si l'on demande aux doctorants de communiquer au sujet de leur thèse, ils concentrent leur propos sur le contenu scientifique et tentent de se contraindre à une présentation normée, comme celle qu'ils ont appris à développer avec leurs pairs : une démarche rigoureuse, des faits, des références appuyées. Ils s'effacent, comme ils en ont pris l'habitude dans leurs communications entre pairs (poster, colloques...) ou dans leurs articles. Le " je » est dissous. Mais, nous l'avons dit, dans les formations et leurs « àcôtés ", dans l'informel, dans la connivence de l'échange de doctorants à doctorants, le 
chaud (re)surgit : les incertitudes, les relations sociales. Le subjectif s'autorise, le « je » se (re)constitue.

Nous choisissons délibérément de laisser la place au « je ", de ne pas effacer l'individu et son vécu. C'est à cette condition que l'expérience de la formation permet de préparer une véritable relation au public de la communication scientifique. Nous faisons alors explicitement la différence entre les faits validés par les pairs et l'expérience personnelle des doctorantes et des doctorants.

Nous ne parlons pas ici de storytelling, autre forme de construction d'une image qui empêche non seulement la relation mais induit une représentation individuelle, de type self-made-man des scientifiques, effaçant la nécessité du collectif, au cœur de nos pratiques de recherche. Nous évitons de re-créer une image d'Épinal de la recherche ou encore des chercheuses et des chercheurs dans un discours de médiation.

Nous ne demandons pas aux jeunes chercheurs - ni aux plus anciens d'ailleurs - d'être des représentants de la "Science ", ni de la sociologie des sciences. Nous leur proposons de parler précisément de là d'où ils parlent. Depuis leur place dans le laboratoire, depuis leur expérience vécue : nous leur demandons de situer leur discours, subjectif et contextualisé, au sein d'une pratique de recherche complexe à laquelle ils participent. Nous les libérons de l'enjeu de la représentation et de la charge d'un discours qui n'est pas le leur. Ils (re)deviennent des témoins subjectifs d'une pratique professionnelle. Ils parlent en leur nom et non « au nom de la science ${ }^{10}$. C'est à cette condition que le lien avec d'autres est possible dans le contexte de la médiation : quand celle ou celui qui parle est bien là, ancré dans son discours, qui devient une parole incarnée.

Comment ce lien est-il rendu possible à partir de ce que les doctorants partagent ? Alors que les objets et les explications scientifiques peuvent très facilement mettre à distance, à l'inverse, le partage du vécu d'une activité professionnelle, faite de ses fragilités et incertitudes, de ses réalités très pragmatiques et concrètes (combien coûte une machine ? Qu'est-ce qu'il se passe quand on interviewe quelqu'un pour une enquête ?...), constituent des prises dont le public se saisit. Ces fragilités ne sont pas l'apanage de la profession de scientifique, il peut donc notamment se créer une reconnaissance interprofessionnelle au-delà de la connaissance scientifique. C'est ce que le public exprime parfois en parlant de "démystification» de l'image du chercheur. Ce rapprochement facilite également la prise de parole et l'expression d'avis, de la part du public, sur l'activité des chercheurs, qui devient plus proche, plus « palpable ». Encore faut-il que les chercheurs acceptent de se prêter au jeu de l'échange, acceptent d'être vulnérables, de poser le "bouclier du savoir » pour discuter, sans relativisme ni positivisme, de ce qui donne sa valeur aux savoirs. Voilà ce à quoi nous formons. 
Speedsearching organisé lors de la Nuit Européenne des Chercheurs à Dijon. Chaque chercheur dialogue durant huit minutes avec une à quatre personnes.

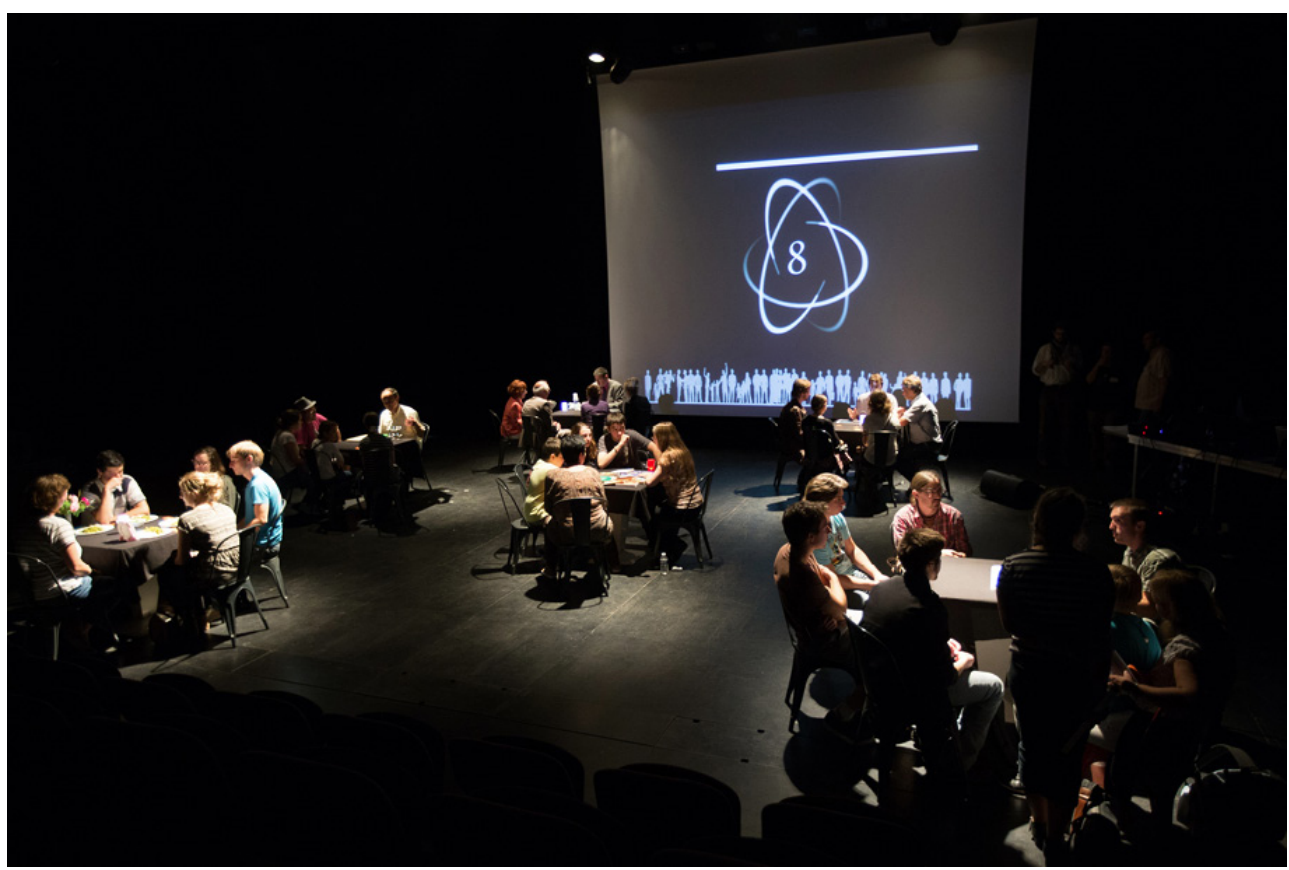

(c) Vincent Arbelet

\section{Du représentant de la science au témoin réflexif d'une pratique professionnelle}

Exprimer les « à-côtés » non marginaux de la science met en mouvement une forme de réflexivité chez les doctorants. Nous l'avons évoqué, ils sont en phase d'initiation professionnelle à la recherche : ce vécu est flou et parfois déstabilisant au regard des parcours jalonnés de réussites scolaires qu'ont souvent pu vivre les doctorants avant d'entrer en thèse. En cours de formation, si la dynamique et l'objectif final sont de s'adresser à l'autre, le doctorant fait l'expérience de la verbalisation, de ce qui - avec des collègues, ou dans le cadre de communications scientifiques spécialisées - était tacite et implicite. Il dit ce qui ailleurs ou « d'habitude » ne se dit pas. Par l'informel, l'expérience peut s'enclencher en douceur. Les discussions s'entourent de la connivence, rassurent et invitent à se libérer d'implicites, de non-dits. Nous reconnaissons presque toujours l'appétit de connaître le vécu de l'autre : "sa relation à son directeur est-elle la même que la mienne? " " comment s'en sort-il avec les expérimentations ? "... L'autre, doctorant ou formateur, n'est pas dans une relation hiérarchique. La discussion se fait en sécurité. Par le temps consacré et l'attention du formateur, ce qui ne pourrait être considéré que comme « juste parler » a les effets d'un travail sur soi une forme de développement professionnel et personnel.

Lors d'un moment de dialogue organisé la veille du festival national des Experimentarium 2016, plusieurs doctorants ont exprimé le besoin de prendre une " bouffée d'air", de " changer de type d'interaction ", de " sortir du compétitif, du regard du directeur ». Lorsqu'on les interroge, les doctorants expriment les effets produits par ces échanges en termes de réconfort et de montée en estime. Jacques parle ainsi de sa première rencontre avec deux formatrices : "je les trouvais très très attentives à ce que 
j'étais et ce que je faisais. Et je me souviens d'avoir parlé beaucoup lors de cet entretien. (...) J'étais pétri d'incertitudes sur le fait que j'avais quelque chose à dire et en même temps, elles étaient hyper convaincues... ».

Cette attention à l'expression personnelle de son vécu de recherche n'est pas abandonnée lorsqu'il s'agit de se former et de s'entraîner à parler au public. S'y être autorisé une première fois, dans l'entre-soi de la formation, en laissant tomber la " cape de l'objectivité » et en reparlant à partir de soi, favorise une communication incarnée dans d'autres situations, quand les autres ne sont plus des pairs mais un " public ». Mais, il faut admettre, à nouveau : les habitudes communicationnelles sont bien ancrées. L'expression publique, même en situation de formation, ramène le doctorant à une posture incorporée dans ses pratiques de communication professionnelle en tant que " chercheur ", en tant que " représentant de la recherche ». S'ils se laissent à nouveau faire (ou " aller ») ils ré-endossent le modèle déficitaire et unilatéral (mimant parfois un faux dialogue). Le " je » est à nouveau effacé. Le phénomène est d'autant plus symptomatique quand il s'agit de passer par l'écrit.

Cette réappropriation du soi dans le discours, par les doctorants (et parvenir à ce qu'ils s'autorisent une parole incarnée dans la communication scientifique) est donc un travail de longue haleine pour le formateur. Ce travail doit ensuite être développé par les doctorants eux-mêmes dans leurs expériences de vulgarisation ou de médiation. Cette réappropriation va à l'encontre d'une science qui serait réduite à ses méthodes scientifiques et résultats (science que les doctorants ont pris le temps d'appréhender pour intégrer la méthodologie rigoureuse inhérente au champ scientifique). Ainsi, le travail de distanciation est important.

La pertinence de cet effort est conditionnée par une réflexion préalable de la part des formateurs : ils s'agit pour nous, formateurs, d'être en accord sur le cadre et le sens de la vulgarisation. Rappelons-le, on peut choisir par exemple entre " diffuser des connaissances " ou " faire appréhender des démarches ». Tout choix est légitime. Le tout est d'avoir conscience du cadre que l'on pose en le faisant. Si l'objectif est de communiquer pour entrer en relation, comme nous l'exposons ici, la logique et la construction des programmes de vulgarisation seront structurées par ce but, notamment autour de l'échange et de la parole des doctorants, au cœur de la formation. Difficile alors de proposer uniquement ou en plus des « manips interactives » dans cette optique. On ne peut pas tout faire en même temps.

Notre choix de formatrice et de formateur implique une attention particulière au vécu des chercheurs qui communiquent. Depuis plus de 10 ans, nous mesurons l'intérêt et les bénéfices qu'il y a à " prendre soin » de ces vulgarisateurs, à penser leur action non comme une tâche à remplir ou une mission assujettie à des gratifications institutionnelles qui la légitimeraient, mais comme une expérience fructueuse et porteuse de sens, en retour, pour l'activité professionnelle et pour l'identité des chercheurs.

En formation, nous veillons donc à entretenir le regard réflexif des doctorants sur euxmêmes : qui parle ? Au nom de quoi, au nom de qui ? Représentent-ils quelque chose d'autre qu'eux-mêmes ? D'où parlent-ils ? À partir de quelle place ? De quels vécus ? Et pour cela, nous partons notamment de l'expérience vécue de la recherche : nous autorisons les doctorants à parler « en leur propre nom ${ }^{11}$, en tant que témoins situés de la recherche d'aujourd'hui ; et nous les invitons à intégrer l'autre (l'autre doctorant, 
puis l'autre en tant que public) dans le discours et l'échange, par intérêt pour ce que cet " autre » peut leur apporter dans la situation de communication, instaurée et partagée.

Nous souhaitons que le doctorant acquiert cette compétence-clé : changer de regard sur ce qu'est une "bonne " communication scientifique et l'envie de considérer la communication comme un mouvement, un cercle vertueux qui enrichira l'un et l'autre par la relation vécue. Une communication incarnée avant d'être efficace.

\section{BIBLIOGRAPHIE}

Faury, M. Parcours de chercheurs. De la pratique de recherche à un discours sur la science : quel rapport identitaire et culturel aux sciences? Thèse, 2012.

Jurdant, B. Parler La Science, Alliage, n59, 2006.

Latour, B. et Woolgar, S. La vie de laboratoire. 1979.

Le Marec, J. Situations de communications dans la pratique de recherche : du terrain aux composites, Études de communication, nำ25, 2002.

Maillot, L. La vulgarisation scientifique et les doctorants : mesure de l'engagement, exploration d'effets pour le chercheur. Thèse, 2018.

Piron, F. Méditation haïtienne. Répondre à la violence séparatrice de l'épistémologie positiviste par l'épistémologie du lien, Sociologie et sociétés, vol. XLIX, n¹, printemps 2017, pp. 33-60.

Stengers, I. Avoir l'étoffe du chercheur, in Une autre science est possible ! 2013, pp. 36-37.

\section{NOTES}

1. L'Ocim propose une cartographie participative des formations de chercheurs et doctorants à la médiation et vulgarisation scientifique en France. http://formation.ocim.fr/cartochercheurs/ 2. "Si le terme "deficit model” émerge en 1996 (diffusé par Irwin et Wynne selon M. Bauer) (...), Baudouin Jurdant, qui s'attaque en 1973 à une théorisation des problèmes de la vulgarisation, expose déjà des caractéristiques que l'on retrouve dans ce modèle : il définit une dissociation, la progression d'une faille, une rupture et il décrit une vulgarisation qui a pour vocation unique ce colmatage » (Maillot, L. p. 39).

3. Voir la thèse de Marine Soichot qui propose un récapitulatif historique de grands courants de la communication publique des sciences et évoque les retours typiques à des postures déficitaires. Soichot, M. Les musées et centres de sciences face au changement climatique. Quelles médiations muséales pour un problème socio-scientifique? 2011.

4. Bodin, C. Espace public et champ scientifique : la publicisation des agents scientifiques sous l'emprise de l'idéologie de la vulgarisation. 2013.

5. Comme le remarquent Besley et al. dans une enquête réalisée auprès de formateurs de stage de communication. Besley, J., Anthony, C., Dudo, D., Yuan, S. and Abi Ghannam, N. Qualitative Interviews with Science Communication Trainers about Communication Objectives and Goals, Science Communication, 38, 2016. 
6. Jurdant, B. Les problèmes théoriques de la vulgarisation scientifique. ULP Strasbourg, réédition des Archives contemporaines, Coll. «Études de Sciences ", 2009.

7. La thèse de Mélodie Faury porte précisément sur l'expérience vécue de la recherche des doctorants, les conflits de normes et de valeurs qu'ils vivent dans leur quotidien du laboratoire, et la relation au directeur de thèse, dans le domaine spécifique de la biologie expérimentale moléculaire.

8. Chao, M. et al. Les expériences de la solitude en doctorat. Fondements et inégalités, SocioLogos, revue de l'association française de Sociologie, $n^{\circ} 10,2015$.

9. Blanchard, A. Comment montrer la science en train de se faire? Du Palais de la découverte à la sociologie des sciences. 2016.

10. Stengers, I. Sciences et pouvoir. 1997, 2002.

11. Dans sa thèse (chapitre 6), Lionel Maillot propose une modélisation de la relation de communication basée sur trois postures communicationnelles (le soi, le chercheur, le présentateur de science) mobilisées lors de situations de vulgarisation ou lors de leur préparation.

\section{RÉSUMÉS}

Les auteurs montrent comment, pour les jeunes chercheuses et chercheurs, les formations doctorales qu'ils organisent, par exemple dans le cadre des actions de l'Experimentarium en Bourgogne ou de la Maison pour la science en Alsace, ne sont pas seulement l'occasion d'acquérir des compétences pour transmettre des messages. Elles sont aussi le lieu de réflexions individuelles et collectives, sources d'émancipation, dans une dynamique de relations incarnées entre sciences et société.

\section{INDEX}

Mots-clés : Formation, doctorants, communication

\section{AUTEURS}

\section{MÉLODIE FAURY}

Chercheuse en études de sciences à l'université de Strasbourg, associée au Lisec équipe Atip. Elle a dirigé la Maison pour la science en Alsace de 2012 à 2018

faury@unistra.fr

\section{LIONEL MAILLOT}

Chercheur en sciences de l'information et de la communication à l'université de Bourgogne, associé au laboratoire Ciméos de l'université de Bourgogne

lionel.maillot@u-bourgogne.fr 